\title{
THE PRESERVATION OF CONVERGENCE OF MEASURABLE FUNCTIONS UNDER COMPOSITION
}

\author{
ROBERT G. BARTLE AND JAMES T. JOICHI ${ }^{1}$
}

Let $f$ be a real-valued measurable function on a measure space $(S, \widetilde{S}, \mu)$ and let $\phi$ be a Borel measurable function of a real variable. Then it is well-known that the composition $\phi \circ f$, defined by $\phi \circ f(s)$ $=\phi(f(s)), s \in S$, is also measurable. Conversely, if $\phi$ is not Borel measurable, then there exists a measurable function $f$ on some measure space such that $\phi \circ f$ is not measurable. We summarize these two remarks in the statement that a function $\phi$ preserves measurability under composition if and only if it is Borel measurable.

The purpose of this note is to characterize functions $\phi$ which preserve convergence (in various senses) of measurable functions. After these results were obtained it was pointed out that Theorems 5 and 6 follow from theorems of P. R. Halmos [2]. Theorem 7 was proved by M. M. Vaŭnberg [3, pp. 204-213], but our proof is much shorter.

1. Preliminary definitions. In the following, $S$ is a set, $\subseteq$ is a $\sigma$-field ( $=\sigma$-algebra) of subsets of $S$, and $\mu$ is a (countably additive extended real-valued non-negative) measure defined on $\widetilde{\Xi}$. We say that a scalar-valued function $f$ is measurable on $(S, \widetilde{S}, \mu)$ if the inverse image under $f$ of every Borel set of scalars belongs to 5 . For simplicity, we shall permit only finite numbers to be taken as values.

We adopt as a standing hypothesis that $\phi$ is a scalar-valued Borel measurable function of a scalar variable. Let $M$ denote a mode of convergence for a sequence of measurable functions. We say that $\phi$ preserves $M$-convergence if, given a sequence $\left(f_{n}\right)$ of measurable functions which is $M$-convergent to $f$ on some measure space, then the sequence $\left(\phi \circ f_{n}\right)$ is $M$-convergent to $\phi \circ f$. Very irregular functions $\phi$ may preserve $M$-convergence of certain sequences or on certain special spaces-what we are interested in is obtaining conditions that will guarantee that $\phi$ preserves the $M$-convergence of every $M$ convergent sequence on any measure space. It is seen that severe restrictions are needed on $\phi$.

2. Convergence of measurable functions. We first discuss almost everywhere convergence on a space of arbitrary measure.

Received by the editors February 8, 1960 and, in revised form, March 3, 1960.

1 The authors are supported in part by grant G-7011 from the National Science Foundation. 
THEOREM 1. The function $\phi$ preserves almost everywhere convergence of sequences of measurable functions if and only if $\phi$ is continuous.

Proof. The sufficiency of the condition is clear. If $\phi$ is not continuous at $x=c$, then there is a sequence $\left(c_{n}\right)$ of scalars converging to $c$ and such that $\left(\phi\left(c_{n}\right)\right)$ does not converge to $\phi(c)$. Let $g_{n}(x) \equiv c_{n}$ and $g(x) \equiv c$. Then $\left(g_{n}\right)$ converges uniformly on $S$ to $g$, but $\left(\phi \circ g_{n}\right)$ does not converge to $\phi \circ g$ at any point.

THEOREM 2. The function $\phi$ preserves uniform convergence, almost uniform convergence, or convergence in measure of sequences of measurable functions if and only if $\phi$ is uniformly continuous.

Proof. If $\phi$ is uniformly continuous and if $|\phi(x)-\phi(y)| \geqq \epsilon$, then $|x-y| \geqq \delta(\epsilon)$. Hence

$$
\begin{aligned}
\left\{s \in S:\left|\phi \circ f_{n}(s)-\phi \circ f(s)\right|\right. & \geqq \epsilon\} \\
& \subseteq\left\{s \in S:\left|f_{n}(s)-f(s)\right| \geqq \delta(\epsilon)\right\} .
\end{aligned}
$$

It follows readily that $\phi$ preserves the stated modes of convergence.

Theorem 1 shows that the continuity of $\phi$ is necessary. Suppose that $\phi$ is not uniformly continuous so that there exist $\epsilon>0$ and sequences $\left(x_{n}\right),\left(y_{n}\right)$ such that $\left|x_{n}-y_{n}\right|<1 / n$ and $\left|\phi\left(x_{n}\right)-\phi\left(y_{n}\right)\right| \geqq \epsilon$. Now let $f$ be defined for $0 \leqq s<\infty$ by $f(s)=x_{k}$ for $k-1 \leqq s<k$ and let $f_{n}$ be defined to equal $y_{n}$ for $n-1 \leqq s<n$ and to equal $f(s)$ otherwise. Then the sequence $\left(f_{n}\right)$ converges uniformly (and hence almost uniformly and in measure) to $f$, but the sequence $\left(\phi \circ f_{n}\right)$ does not converge in measure (and hence does not converge uniformly or almost uniformly) to $\phi \circ f$.

3. Convergence of totally measurable functions. As in [1, III.2.10], we say that a scalar-valued function $f$, defined on $S$, is totally measura$b l e^{2}$ on $(S, \mathfrak{S}, \mu)$ if it is the limit in measure (or almost uniformly) of a sequence of $\widetilde{S}$-simple functions. On a space of finite measure, a function is measurable if and only if it is totally measurable. A totally measurable function has the property that given $\epsilon>0$, there is a set $E$ in $\subseteq$ with $\mu(E)<\epsilon$ such that $f$ is bounded on $S-E$.

It is proved in [1, III.2.12] that if $\phi$ is continuous and $f$ is totally measurable, then $\phi \circ f$ is totally measurable. The key to the argument is that $f$ is nearly bounded and that $\phi$ is uniformly continuous on most of the range of $f$.

2 Our use of the terms "measurable" and "totally measurable" differ slightly from that in [1], but should not cause any confusion to the reader as we restrict our attention to the case of a countably additive measure. 
THEOREM 3. The function $\phi$ preserves almost everywhere convergence, almost uniform convergence, or convergence in measure of sequences of totally measurable functions if and only if $\phi$ is continuous.

Proof. The necessity of the condition has already been seen. The sufficiency of the condition for convergence in measure is stated without proof in [1, III.2.12]. It can be proved by using the argument sketched in the preceding paragraph. The other modes of convergence are handled similarly.

Theorem 4. A function $\phi$ preserves uniform convergence of sequences of totally measurable functions if and only if it is uniformly continuous.

The proof of the necessity is similar to that of Theorem 2 and will be omitted.

Since measurability coincides with total measurability on spaces of finite measure, we note that the criterion for the preservation of almost uniform convergence and convergence in measure is different on spaces of finite and infinite measure.

4. Convergence in $L_{p}$. We now examine when $\phi$ preserves convergence in one of the spaces $L_{p}, 1 \leqq p \leqq \infty$. It is clear that it is necessary that $\phi$ be continuous. Also, in the case of infinite measure, we must have $\phi(0)=0$. We first find conditions on a continuous function $\phi$ to preserve membership in $L_{p}, 1 \leqq p<\infty$. We shall treat the case of finite measure first.

THEOREM 5. Let $\phi$ be a continuous scalar-valued function and let $1 \leqq p<\infty$. Then $\phi \circ f$ is in $L_{p}$ over a space of finite measure whenever $f$ is in $L_{p}$ if and only if

$$
\limsup _{|x| \rightarrow \infty}\left|\frac{(\phi x)}{x}\right|<\infty \text {. }
$$

Proof. If $(*)$ is satisfied, then since $\phi$ is continuous, there is a constant $K>0$ such that $|\phi(x)| \leqq K(|x|+1)$ for all $x$. If $f$ is in $L_{p}(S, \mathfrak{S}, \mu)$ and $\mu(S)$ is finite, then $\phi \circ f \in L_{p}$.

Conversely, if $(*)$ does not hold, then there is a sequence $\left(v_{n}\right)$ with $\left|v_{n}\right| \rightarrow \infty$ such that $\left|\phi\left(v_{n}\right)\right|=n\left|v_{n}\right|$ for all sufficiently large integers $n$. For simplicity in notation suppose this relation holds for all $n$ and that $v_{n} \neq 0$. Let $a_{n}=1 /\left(n^{2}\left|v_{n}\right|^{p}\right)$, so that $A=\sum a_{n}$ converges. Let $A_{0}=0$ and $A_{n}=a_{1}+\cdots+a_{n}$ for $n>0$, and define $f$ on $0 \leqq s \leqq A$ by

$$
f(s)=v_{n}, \quad A_{n-1} \leqq s<A_{n}, \quad n=1,2, \cdots .
$$

It is clear that $f$ is Borel measurable and easily seen that $f$ is in $L_{p}(0, A)$, while $\phi \circ f$ is not in $L_{p}(0, A)$. 
Corollary 1. If $\phi$ is continuous, condition (*) is satisfied if and only if there is a constant $K$ such that $|\phi(x)| \leqq K|x|$ for all sufficiently large $x$.

COROLlaRY 2. The condition of the theorem is satisfied if $\phi$ is either bounded or uniformly continuous.

Proof. The bounded case is clear. If $\phi$ is uniformly continuous there are positive numbers $\epsilon, \delta$ such that $|\phi(x)| \leqq(\epsilon / \delta)|x|$ $+(\epsilon+|\phi(0)|)$. Hence the condition of Corollary 1 holds for large $x$.

Theorem 5 fails for a space of infinite measure, where it is necessary to add a condition at $x=0$. The proof of the next theorem is similar to that of Theorem 5 .

THEOREM 6. Let $\phi$ be a continuous scalar-valued function and let $1 \leqq p<\infty$. Then $\phi \circ f$ is in $L_{p}$ whenever $f$ is in $L_{p}$ if and only if there is a positive number $K$ such that $|\phi(x)| \leqq K|x|$ for all $x$.

CoROllary. If $\phi$ is continuous, then the condition of the theorem is satisfied if and only if

$$
\limsup _{|x| \rightarrow 0}\left|\frac{\phi(x)}{x}\right|<\infty, \quad \quad \limsup _{|x| \rightarrow \infty}\left|\frac{\phi(x)}{x}\right|<\infty .
$$

Proof. The uniform continuity of $\phi$ for $1 / M \leqq|x| \leqq M$ gives an estimate of the form $|\phi(x)| \leqq(\epsilon / \delta)|x|+\epsilon$. The remaining hypotheses enable one to show that $|\phi(x)| \leqq K|x|$ for all $x$.

The condition assuring that $\phi$ map $L_{p}$ into $L_{p}$ also assures that $\phi$ preserves $L_{p}$-convergence.

Theorem 7. Let $p$ satisfy $1 \leqq p<\infty$. A function $\phi$ preserves $L_{p^{-}}$ convergence under composition if and only if $\phi$ is continuous and $|\phi(x)| \leqq K|x|$ for some $K$ and all $x$.

Proof. The sufficiency of the condition follows from Theorem III.3.6 and III.9.11 of [1] and will not be given in detail.

Conversely, suppose the inequality is not satisfied and choose a sequence of scalars $z_{n} \neq 0$ such that $\left|\phi\left(z_{n}\right)\right|=n\left|z_{n}\right|$. Let $b_{n}=1 /\left(n\left|z_{n}\right|^{p}\right)$ and define $f_{n}$ to equal $z_{n}$ if $0 \leqq s \leqq b_{n}$ and to equal 0 otherwise. The sequence $\left(f_{n}\right)$ converges to the zero function $\theta$ in $L_{p}$, but $\left(\phi \circ f_{n}\right)$ does not converge to $\phi \circ \theta$ in $L_{p}$.

TheORem 8. Let $p$ satisfy $1 \leqq p<\infty$. A function $\phi$ preserves $L_{p^{-}}$ convergence on spaces of finite measure if and only if $\phi$ is continuous and satisfies either 


$$
\begin{gathered}
\limsup _{|x| \rightarrow \infty}\left|\frac{\phi(x)}{x}\right|<\infty \text {, or } \\
\text { for some } K>0,|\phi(x)| \leqq K|x| \text { for all } x \text { with }|x| \geqq K .
\end{gathered}
$$

We omit the proof of this result.

It remains to treat the case $p=\infty$. The proof of the next theorem is direct.

TheORem 9. A Borel measurable function $\phi$ has the property that $\phi \circ f$ is in $L_{\infty}$ whenever $f$ is in $L_{\infty}$ if and only if $\phi$ maps bounded sets into bounded sets.

THEOREM 10. The function $\phi$ preserves $L_{\infty}$-convergence if and only if it is continuous.

Proof. The necessity of the condition is clear.

Since a convergent sequence in $L_{\infty}$ is essentially uniformly bounded and $\phi$ is uniformly continuous on bounded sets, the sufficiency is readily proved.

\section{REFERENCES}

1. N. Dunford and J. T. Schwartz, Linear operators, Part I, New York, Interscience, 1958.

2. P. R. Halmos, Functions of integrable functions, J. Indian Math. Soc. vol. 11 (1947) pp. 81-84.

3. M. M. Valnberg, Variational methods in the investigation of non-linear operators, Moscow, Gosudarstv. Izdat. Tehn.-Teor. Lit., 1956 (Russian).

UNIVERSITY OF ILLINOIS 